\title{
Avaliação nutricional, consumo alimentar e frequência de ultraprocessados em escolares da rede pública
}

\author{
Nutritional evaluation, food consumption and frequency of ultraprocessed in public schools \\ Evaluación nutricional, consumo de alimentos y frecuencia de ultraprocesos en escuelas \\ de la red pública
}

Liejy Agnes dos Santos Raposo Landim ${ }^{1}$, Maraysa Carvalho Cordeiro ${ }^{*}$, Amanda Marreiro Barbosa ${ }^{2}$, Juliana Soares Severo², Daniela Fortes Neves Ibiapina', Brenda Aparecida Diogo Pereira'1.

\section{RESUMO}

Objetivo: Avaliar a ingestão de alimentos ultraprocessados e sua importância no estado nutricional em crianças de um colégio de rede pública de Teresina-PI. Métodos: Trata-se de análise observacional transversal, investigação de campo descritiva, exploratória, quantitativa, realizada com 105 crianças de 7 a 10 anos, de ambos os sexos. A pesquisa foi aprovada através do Comitê de Ética em Pesquisa. Foram coletados dados antropométricos, analisados dois recordatórios de 24 horas para averiguação do consumo de alimentos ultraprocessados, utilizando o programa Dietbox. Para análise dos outros dados, procedeu-se com análise descritiva. Resultados: Avaliados 105 alunos, 18,1\% (19) estão com sobrepeso, 7,6\% (8) com obesidade e 5,7\% (6) com obesidade grave, 2,9\% (03) com magreza e 2,9\% (03) com magreza acentuada. Os macronutrientes foram distribuídos em médias ficando carboidratos $(67,0 \pm 175,9)$, lipídios $(31,9 \pm 6,4)$ e proteína $(18,19 \pm 4,4)$. No consumo de ultraprocessados se destacam os sucos industrializados com $44 \%$ (46), em seguida embutidos com $43 \%$ (45), bolos e biscoitos doces com $42 \%$ (44). Conclusão: Os escolares apresentaram desenvolvimento de sobrepeso, obesidade e excesso de gordura abdominal possuindo relação com riscos de doenças cardiovasculares, juntamente com elevado consumo de ultraprocessados.

Palavras-chave: Escolares, Avaliação nutricional, Alimentação, Industrializados.

\begin{abstract}
Objective: To evaluate the intake of ultra-processed foods and their importance in the nutritional status of children at a public school in Teresina-PI. Methods: This is a cross-sectional observational analysis, descriptive, exploratory, quantitative field investigation, carried out with 105 children aged 7 to 10 years, of both sexes. The research was approved by the Research Ethics Committee. Anthropometric data were collected and two 24-hour reminders were analyzed to ascertain the consumption of ultra-processed foods, using the Dietbox program. For analysis of other data, we proceeded with descriptive analysis. Results: Evaluated 105 students, $18.1 \%$ (19) are overweight, $7.6 \%$ (8) with obesity and $5.7 \%$ (6) with severe obesity, $2.9 \%(03)$ with thinness and 2, 9\% (03) with marked thinness. Macronutrients were distributed as means being carbohydrates $(67.0 \pm 175.9)$, lipids $(31.9 \pm 6.4)$ and protein $(18.19 \pm 4.4)$. In the consumption of ultraprocessed foods, $44 \%(46)$, followed by inlays with $43 \%(45)$, cakes and sweet biscuits with $42 \%$ (44) stand out. Conclusion: The students had development of overweight, obesity and excess abdominal fat, being related to the risk of cardiovascular diseases, together with high consumption of ultra-processed foods.
\end{abstract}

Keywords: Students, Nutritional assessment, Diet, Industrialized.

\section{RESUMEN}

Objetivo: Evaluar la ingesta de alimentos ultraprocesados y su importancia en el estado nutricional de los niños en una escuela pública en Teresina-PI. Métodos: Este es un análisis de observación transversal, descriptivo, exploratorio, investigación de campo cuantitativa, realizado con 105 niños de 7 a 10 años, de ambos sexos. La investigación fue aprobada por el Comité de Ética de Investigación. Se recopilaron datos

1 Centro Universitário Santo Agostinho (UNIFSA), Teresina-PI. *E-mail: maraysacarvalho_@hotmail.com

2 Universidade Federal do Piauí (UFPI), Teresina-PI. 
antropométricos, se analizaron dos retiros del mercado de 24 horas para determinar el consumo de alimentos ultraprocesados, utilizando el programa Dietbox. Para el análisis de otros datos, se procedió al análisis descriptivo. Resultados: Evaluado 105 estudiantes, 18.1\% (19) tienen sobrepeso, 7.6\% (8) con obesidad y $5.7 \%$ (6) con obesidad severa, 2.9\% (03) con delgadez y 2, 9\% (03) con marcada delgadez. Los macronutrientes se distribuyeron como carbohidratos (67.0 \pm 175.9$)$, lípidos (31.9 \pm 6.4$)$ y proteínas (18.19 \pm 4.4). En el consumo de alimentos ultraprocesados, destacan el $44 \%$ (46), seguido de las incrustaciones con el $43 \%$ (45), los pasteles y las galletas dulces con el 42\% (44). Conclusión: los estudiantes desarrollaron sobrepeso, obesidad y exceso de grasa abdominal, estando relacionados con el riesgo de enfermedades cardiovasculares, junto con un alto consumo de alimentos ultraprocesados.

Palabras clave: Estudiantes, Evaluación nutricional, Alimentación, Industrializada.

\section{INTRODUÇÃO}

A alimentação saudável é um indicativo de mais saúde e qualidade de vida, com diminuição do risco de doenças e aumento da imunidade, com geração de energia e redução do cansaço físico e mental. A criança se encontra em uma fase e que é necessária uma atenção especial, pois está em processo de crescimento físico, em desenvolvimento acelerado com ganho de massa magra e óssea, com amadurecimento de seu sistema imunológico, gerando, assim, uma necessidade de uma nutrição adequada, que ofereça energia e nutrientes necessários para seu bom crescimento e desenvolvimento saudável. Caso isso não aconteça, pode ocasionar um déficit ou um excedente nutricional, gerando complicações como sobrepeso, obesidade ou uma magreza acentuada, além de problemas com baixa estatura (BRASIL, 2013).

Segundo Soares LR et al. (2013), uma má alimentação, com a ingestão de alimentos de alta densidade calórica e consumo de alimentos ultraprocessados, conjugado a redução da atividade física, ou sedentarismo, gera danos e inúmeros prejuízos à saúde, como a obesidade e sobrepeso, e possível surgimento de doenças crônicas não transmissíveis. Com isso, são preocupantes o aumento dessas taxas no Brasil, sobretudo, em crianças e adolescentes.

Durante a infância, crianças passam maior parte do tempo nas escolas, local que possui o papel de estimular hábitos saudáveis, que serão levados para a vida adulta. Fazendo parte deste processo, o Programa Nacional de Alimentação Escolar (PNAE) possui o objetivo de fornecer uma alimentação escolar saudável durante o período de permanência do aluno na escola, contemplando também ações de Educação Alimentar e Nutricional (BRASIL, 2013).

Essas crianças fazem a maioria de suas refeições diárias nas escolas, em especial as que estudam em rede pública de ensino, pois a situação econômica das famílias, e de pobreza, limitam a alimentação adequada e saudável para seus filhos, havendo substituição de frutas por doces e salgadinhos gordurosos e industrializados, ocasionando níveis altos de obesidade e subnutrição.

O aumento da ingestão de alimentos ultraprocessados juntamente a elevada quantidade energética, contendo carboidratos de alto índice glicêmico, gorduras saturadas, junto a maiores concentrações de sódio, menos fibras, vitaminas e minerais, está interferindo de forma negativa a evolução e a condição nutricional das crianças (SANTOS DM, 2017; BERNARDI JR, et al. 2010). Diante disso, o presente estudo teve como objetivo avaliar a prevalência do consumo de alimentos ultraprocessados e seus fatores associados ao estado nutricional entre escolares de rede pública.

\section{MÉTODOS}

Trata-se de uma análise observacional, transversal, uma investigação de campo descritiva, exploratória, quantitativa, na qual foram avaliados escolares de 7 a 10 anos, matriculados do $1^{\circ}$ ao $4^{\circ}$ ano em uma escola pública localizada na zona urbana, na cidade de Teresina (PI).

A definição da amostra foi baseada no número de alunos matriculados regularmente (241 crianças). Dessas crianças, as que se encontram na faixa etária do presente estudo (7 a 10 anos) foram 149 crianças. Para definição da quantidade amostral foi empregado o aplicativo para cálculo amostral, a calculadora online, aderindo o nível de significância de $5 \%$ de possibilidade, permanecendo o número amostral a ser avaliada 
no presente estudo de 105 crianças de ambos os sexos. Foram incluídos na análise, aqueles escolares que aceitaram participar, bem como, foram autorizados por seus pais ou responsáveis legais a participar do estudo, por meio da assinatura de um Termo de Consentimento Livre e Esclarecido (TCLE) e Termo de Assentimento Livre e Esclarecido (TALE). A coleta de dados ocorreu no decurso de maio do ano de 2019.

A pesquisa foi aprovada através do Comitê de Ética em Pesquisa- CEP do Centro Universitário Santo Agostinho (UNIFSA), tendo com número do parecer 3.158.930 e CAEE de número 03304518.1.0000.5602. Diante disso, foi esclarecida a natureza do estudo, com os pesquisadores se comprometendo ao sigilo e à total descrição quanto aos dados coletados.

Os participantes da pesquisa foram selecionados de acordo com os seguintes critérios de inclusão: todas as crianças da amostra deveriam estar matriculadas regularmente na escola pesquisada, apresentando idade entre 7 e 10 anos, de ambos os sexos, não apresentando diagnóstico clínico de patologias crônicas e diagnóstico nutricional grave.

Como critérios de exclusão, não puderam participar todas as crianças que apresentassem doenças crônicas endócrinas que comprometem seu estado nutricional, portadoras de alguma deficiência física, problemas cognitivos ou dificuldade em falar ou escrever.

Os dados utilizados na avaliação antropométrica foram: o peso $(\mathrm{kg})$ através de balança portátil digital onrom HN, com carga mínima de $1 \mathrm{~kg}$ e máxima de 150k; a estatura, através de fita métrica, inelástica, fixada na parede em linha reta. Os integrantes foram avaliados descalços, com os braços dispostos ao lado do corpo, em coloção ereta.

A análise dos parâmetros (Peso, Estatura e IMC) foi utilizada a Caderneta de Saúde da Criança, segundo propõe a Organização Mundial de Saúde - OMS, 2007. Tendo como base os gráficos seguindo uma escala de escores, P/I (peso para idade), E/I (Estatura para Idade) e IMC/I (Índice de Massa Corpórea para idade) (MINISTERIO DA SAÚDE, 2013). Para a classificação do estado nutricional foi realizada através do Índice de Massa Corporal para a idade (IMC/I) calculado a partir do peso $(\mathrm{kg})$ do participante do estudo dividido por sua estatura (cm) elevada ao quadrado (OMS, 2007).

A aferição da circunferência da cintura teve conforme referência Freedman DS, et al. (1999), de acordo com o sexo e a faixa etária e foi realizada com os participantes em pé, utilizando uma fita métrica não elástica, milimetrada em centímetros, flexível e não extensível, com precisão de 0,1 centímetros, circundando a linha natural da cintura, na região mais estreita entre o tórax e o quadril, no ponto médio entre a última costela e a crista ilíaca. Os participantes estavam em posição ereta, com abdômen relaxado, braços estendidos ao longo do corpo e pés afastados um do outro.

Com relação as variáveis referentes aos valores energéticos coletados: percentual de carboidratos, proteínas e lipídios na qual, foram coletadas em 2 dias e realizou-se uma média aritmética referente ao valor alcançado. Foram realizados dois recordatórios alimentares de 24 horas (R24h), para a avaliação do consumo de alimentos ultraprocessados, aplicados em dias consecutivos. No instante da entrega dos recordatórios foram orientadas detalhadamente para as crianças a forma correta como o R24h deveria ser preenchido, como registrar os alimentos, como diferenciar os tipos de refeições, quantidades, medidas caseiras e horários em que as mesmas consumiram.

Os recordatórios foram entregues às pesquisadoras posteriormente dois dias, com esclarecimentos e ajustes de informações sobre o que a criança relatou ter ingerido. Em seguida foi realizada a análise com base nos valores de referências das DRIs - Dietary Referência Intakes, para os macronutrientes (Carboidratos 55 a 60\%); (Lipídios 25 a 30\%); (Proteína 15 a 25\%).

Para a análise do consumo de ultraprocessados foi aferida de acordo com as repetições que esses alimentos eram descritos nos recordatórios de 24 horas e sua significância em percentual, utilizando o programa Dietbox para análise do valor energético e de macronutrientes.

Os dados foram organizados em planilhas do Excel® (2016) para realização de análise descritiva das variáveis observadas. Os resultados obtidos foram organizados em forma de tabelas e os parâmetros antropométricos forma analisados individualmente (peso, altura, circunferência da cintura e IMC), obtendo-se a média geral de cada turma em relação a esses parâmetros. 


\section{RESULTADOS E DISCUSSÃO}

Conforme a Tabela 1, foram avaliadas 105 crianças, com idade média de $8,5 \pm 1,29$ anos, sendo 50,5\% do gênero masculino (53) e 49,5\% do gênero feminino (52). Cuja média obtida para as crianças foi de $32,2 \mathrm{~kg}$ $\pm 7,86$ para o peso; $135,0 \mathrm{~cm} \pm 0,06$ para altura; $17,7 \mathrm{~kg} / \mathrm{m}^{2} \pm 3,42$ para IMC e $60 \mathrm{~cm} \pm 8$ para circunferência da cintura.

Foi possível observar que 1,9\% $(n=2)$ dos escolares estão com altura abaixo do recomendado, não podendo ser caracterizada como anormal, pois podem representar alunos com estatura familiar reduzida. As características da população estudada encontram-se na Tabela 1 abaixo.

Tabela 1 - Distribuição da amostra segundo os parâmetros antropométricos e classificação do estado nutricional de escolares de rede pública, Teresina-PI, 2019.

\begin{tabular}{|c|c|c|}
\hline Variáveis & Total $(\mathbf{n})$ & Total (\%) \\
\hline Sexo & 105 & $(100 \%)$ \\
\hline Masculino & 53 & $(50,5 \%)$ \\
\hline Feminino & 52 & $(49,5 \%)$ \\
\hline Indicadores & $\mathbf{N}$ & $\%$ \\
\hline \multicolumn{3}{|l|}{$\mathbf{P} / \mathbf{I}$} \\
\hline Peso elevado & 15 & 14,3 \\
\hline Peso adequado & 86 & 81,9 \\
\hline Peso baixo & 4 & 3,8 \\
\hline \multicolumn{3}{|l|}{$E / I$} \\
\hline Estatura elevada & 1 & 1 \\
\hline Estatura adequada & 102 & 97,1 \\
\hline Estatura baixa & 2 & 1,9 \\
\hline \multicolumn{3}{|l|}{ IMC/I } \\
\hline Eutrofia & 66 & 62,8 \\
\hline Obesidade & 8 & 7,6 \\
\hline Obesidade grave & 6 & 5,7 \\
\hline Sobrepeso & 19 & 18,1 \\
\hline Magreza & 3 & 2,9 \\
\hline Magreza acentuada & 3 & 2,9 \\
\hline \multicolumn{3}{|l|}{ C.C } \\
\hline Com excesso de adiposidade & 11 & 10,5 \\
\hline Sem excesso de adiposidade & 94 & 89,5 \\
\hline Variáveis & \multicolumn{2}{|r|}{ Média/desvio padrão } \\
\hline Idade, anos $(n=105)$ & \multicolumn{2}{|r|}{$8,5 \pm 1,29$} \\
\hline Peso, $\mathrm{kg}(\mathrm{n}=105)$ & \multicolumn{2}{|r|}{$32,2 \pm 7,86$} \\
\hline Estatura, $\mathrm{cm}(\mathrm{n}=105)$ & \multicolumn{2}{|r|}{$135,0 \pm 0,06$} \\
\hline IMC, $\mathrm{kg} / \mathrm{m}^{2}(\mathrm{n}=105)$ & \multicolumn{2}{|r|}{$17,7 \pm 3,42$} \\
\hline C.C $(n=105)$ & \multicolumn{2}{|r|}{$60 \pm 8$} \\
\hline
\end{tabular}

Legenda: P/I: Peso para a Idade. E/I: Estatura para a Idade. IMC/I: Índice de Massa Corporal para a Idade. C.C: Circunferência da Cintura. Fonte: Cordeiro MA, et al., 2019. 
Os resultados expressos em média, frequência \% (percentual) e \pm DP (Desvio Padrão). No que se refere ao IMC, os resultados da Tabela 1 indicam que 18,1\% $(n=19)$ dos escolares apresentam sobrepeso, 7,6\% $(n=8)$ estão com obesidade e 5,7\% ( $n=6)$ com obesidade grave. De acordo com Miranda JMQ, et al. (2015), a obesidade tem sido caracterizada pelo aumento do tecido adiposo e do peso corporal, com tratamento altamente complexo. Seu estudo também foi realizado com estudantes de escola pública, no qual participaram 107 crianças, sendo $22,5 \%$ (45) do sexo masculino e $31 \%$ (62) do sexo feminino.

Os resultados verificados pelos autores corroboraram com o presente estudo uma vez que foram identificados que no grupo de crianças de 8 anos do sexo masculino estavam classificados com obesidade e entre os grupos nas faixas etárias de 9 e 10 anos apresentaram-se com sobrepeso, os autores associaram esse quadro de sobrepeso e obesidade infantil ao fator socioeconômico, uma vez que pode desencadear o desenvolvimento de doenças crônicas. Destaca-se o percentual de 2,9\% (03) de crianças participantes classificadas com magreza, igualmente ao percentual de 2,9\% (03) de crianças com magreza acentuada. Isso demonstra que a má alimentação não gera apenas um excedente de peso, mas, em contrapartida, também pode provocar um déficit nutricional, igualmente preocupante.

Guedes D, et al. (2013) mostram resultados semelhantes ao presente estudo, pois encontraram valores equivalentes quanto ao IMC, com prevalência de baixo peso corporal/magreza, sobrepeso e obesidade, em amostra representativa de crianças e adolescentes assistidos pelo Programa Segundo Tempo do Ministério de Esporte. Ainda sobre seu estudo, revelam que evidências sugerem a desnutrição em idades mais baixas, o que pode acarretar problemas no crescimento físico e no desenvolvimento cognitivo, favorece o aparecimento de doenças infectocontagiosas, limitando a capacidade física e gerando, na idade adulta, elevados riscos para doenças degenerativas.Chaves C et al. (2013) abordaram a relação peso/idade, percebendo que $20,4 \%$ de sua amostra estava com peso abaixo do recomendado, $4,5 \%$ das crianças tiveram classificação de peso muito baixo para a idade e $2,3 \%$ foram incluídas na faixa de sobrepeso.

Foi evidenciada estatura baixa para a idade em $27,3 \%$ das crianças, a qual prevaleceu na fase pré-escolar. Além disso, observaram que, das 37 crianças que se encontraram na faixa de normalidade no percentil peso/estatura, 11 apresentaram déficit estatural. Esses percentuais se assemelham apenas nos valores encontrados para crianças com peso muito baixo para idade no nosso estudo, divergindo com os resultados encontrados quanto ao percentual de crianças com sobrepeso, que no presente estudo foi de $18,1 \%$.

Com relação à circunferência da cintura, seguindo o parâmetro de Freedman DS, et al. (1999), 10,5\% (11) dos escolares avaliados apresentaram excesso de adiposidade abdominal, evidenciando um alto risco para doenças cardiovasculares, deixando em alerta a probabilidade de crianças tão jovens terem a chance de desenvolver diabetes, pressão alta, colesterol em níveis inadequados e possíveis desordens celulares, acarretando cânceres ou problemas relacionados a alergias e intoxicações graves.

Diante desses resultados, é possível revelar que esse grupo populacional possui um índice de sobrepeso e obesidade maior em relação a níveis de magreza e desnutrição, dados que corroboram com a teoria de Rocha LM, (2013), a qual considera que as tendências da desnutrição e obesidade são características do processo de transição nutricional do país, pois, ao mesmo tempo em que as taxas de desnutrição declinam em crianças, cresce em ritmo acelerado a prevalência de sobrepeso e obesidade infantil, tendo como um dos fatores o aporte energético ingerido por essas crianças com distribuição de macronutrientres (carboidratos, proteínas e lipídios) em percentuais desproporcionais a suas necessidades diárias, dados apresentados na Tabela 2.

Tabela 2 - Média e desvio padrão do aporte energético e macronutrientes dos escolares de rede pública, Teresina-PI, 2019.

\begin{tabular}{ccc}
\hline VET & Média(kcal/\%) $\pm \mathbf{D P}$ & Média(kcal/\%) Ideais \\
\hline VET encontrado & $1661,6 \pm 527,3$ & $1.736 \mathrm{kcal}$ \\
Carboidrato & $67,0 \pm 175,9$ & $55-60$ \\
Lipídio & $31,9 \pm 6,4$ & $25-30$ \\
Proteína & $18,19 \pm 4,4$ & $15-25$
\end{tabular}

Legenda: VET: Valor Energético Total. Fonte: Cordeiro MA, et al., 2019. 
Os resultados expressos em média kcal (quilocalorias) e frequência \% (percentual) \pm DP (Desvio Padrão). De acordo com a tabela acima, é possível observar que a média do consumo do valor energético total (VET) encontrado $(1661,6 \pm 527,3)$, com distribuição entre os macronutrientes equivalente em médias de carboidratos $(67,0 \pm 175,9)$, lipídios $(31,9 \pm 6,4)$ e proteína $(18,19 \pm 4,4)$. Diante destes resultados, percebese que o aporte calórico dessas crianças é constituído, em sua maioria, por carboidratos e gorduras, conseguindo levar à compreensão dos dados já apresentados na Tabela 1, pois esses escolares apresentaram altos índices de sobrepeso e obesidade.

Esse consumo é superior ao preconizado na DRIs - Dietary (2002), que recomenda que o consumo de carboidratos seja em média de 55 a $60 \%$ do VET, e lipídios sejam de 25 a 30\%, evidenciando que o único macronutriente consumido em de acordo com as recomendações é a proteína, que possui como valores de referência de 15 a $25 \%$ para o VET diário. Desse modo, a dieta da amostra analisada é, em sua maioria, hiperglicídica, hiperlipídica e normoproteica.

Conforme as DRIs (2002), a necessidade nutricional representa o nível mínimo de ingestão de energia e nutrientes capaz de atender às necessidades fisiológicas de grupos distintos, considerando uma margem de segurança que ultrapasse a necessidade básica (FIDELIS CMF e OSÓRIO MF, 2007).

SILVA J et al. (2010) realizou um estudo cujo resultados demonstraram que, dos indivíduos avaliados quanto à ingestão energética, 5,6\% estavam com ingestão excessiva de energia, o que pode facilitar o sobrepeso e, desta forma, tornar-se um fator de risco para doenças, em especial, de origem metabólica na vida adulta. Em relação à excessiva ingestão de energia, esse resultado é semelhante ao nosso estudo.

Há uma grande preocupação com a qualidade e composição desses alimentos, que estão sendo consumidos em quantidades elevadas pelos escolares, gerando sobrepeso e obesidade com carências nutricionais, de vitaminas e minerais necessários ao bom crescimento e funcionamento do organismo.

Os alimentos que fazem parte do consumo diário dessas crianças, e que estão aumentando o percentual de carboidratos e gorduras das mesmas, estão apresentados na Tabela 3, fazendo destaque ao elevado consumo de produtos ultraprocessados.

Tabela 3 - Média e percentual do consumo de alimentos ultraprocessados de crianças de uma escola da rede pública, Teresina-PI, 2019.

\begin{tabular}{cccc}
\hline Variável & $\mathbf{n}$ & $\%$ & $\mathbf{n}$ \\
\hline Alimentos & $\begin{array}{c}\text { Quantidade de } \\
\text { crianças que } \\
\text { consomem }\end{array}$ & $\begin{array}{c}\% \text { de } \\
\text { crianças }\end{array}$ & $\begin{array}{c}\text { Número de vezes que } \\
\text { se repete no } \\
\text { recordatórios }\end{array}$ \\
\hline Doces & 12 & $11 \%$ & 17 \\
Refrigerantes & 34 & $32 \%$ & 52 \\
Biscoitos recheados & 40 & $38 \%$ & 63 \\
Suco industrializados & 46 & $44 \%$ & 71 \\
Achocolatado em pó & 33 & $31 \%$ & 61 \\
Embutidos & 45 & $43 \%$ & 80 \\
Bolos e Biscoitos doces & 44 & $42 \%$ & 78 \\
Salgadinhos e pipoca industrializada & 23 & $22 \%$ & 77 \\
Biscoitos salgados & 30 & $29 \%$ & 41 \\
Bebidas achocolatadas & 17 & $16 \%$ & 25 \\
Salgados e batata frita & 38 & $36 \%$ & 47 \\
Bebidas lacta & 35 & $33 \%$ & 53 \\
Farinha láctea & 1 & $1 \%$ & 1 \\
Leite em pó & 43 & $41 \%$ & 101 \\
Nissin miojo & 8 & $8 \%$ & 10 \\
\hline Total & 105 & - & - \\
\hline
\end{tabular}

Fonte: Cordeiro MA, et al., 2019. 
A Tabela 3 apresenta os ultraprocessados citados nos recordatório de 24 horas dos escolares, indicando a quantidade de crianças que consomem cada produto alimentício. Os ultraprocessados mais consumidos foram os sucos industrializados, com $44 \%$ (46) crianças referindo consumo. Em seguida, $43 \%$ (45) dos escolares mencionaram os embutidos, $42 \%$ (44) mencionaram bolos e biscoitos doces e $38 \%$ (40) mencionaram biscoitos recheados.

Considerando a quantidade de vezes em que esses produtos alimentícios foram citados nos recordatórios pelas crianças que referiam consumi-los, entre os itens listados, os que obtiveram mais menções sobre 0 consumo foram os embutidos, com 80 repetições, bem próximo das 78 vezes em que os bolos e biscoitos doces foram citados, seguidos pelos sucos industrializados, com 71 vezes menções e, por fim, os biscoitos recheados, com 63 recorrências.

Os dados da tabela acima revelam o elevado consumo de alimentos ultraprocessados, o que é preocupante. De acordo com os estudos de Adami FS e Conde RS, (2016), esses ultraprocessados são ricos em aditivos químicos como emulsificantes, estabilizantes, conservantes, corantes, edulcorantes, aromatizantes, realçadores de sabor e estabilizantes de cor.

Apesar do rigoroso processo que leva à permissão do uso de tais aditivos nos produtos ultraprocessados, essas substâncias trazem prejuízos ao organismo humano. Diante dos dados expostos nas tabelas 1 e 2 , é possível perceber que os escolares estão com excesso de adiposidade abdominal, sobrepeso e obesidade, como reflexo de uma alimentação excedente em carboidratos e lipídios, convergindo com os dados da Tabela 3, que mostra que este excesso tem como base o alto consumo de alimentos ultraprocessados.

Os embutidos se destacam como o produto com maior número de menções nos recordatórios, sendo itens alimentícios ricos em nitrito e nitrato, que são sais com função de conservar, agregar cor e sabor. No entanto, após o consumo de tais substâncias, ocorre no trato digestório a produção de $\mathrm{N}$-nitrosos e nitrosaminas, composto que, quando consumidos em altas quantidades, produz no organismo características tóxicas, cancerígenas, mutagênicas, teratogênicas e metemoglobinemia (ARAÚJO PF e RODRIGUES RS, 2008; DUTRA CB, RATH S e REYES FGR, 2007; GUERREIRO RS, SÁ MS e RODRIGUES LAP, 2012; OLIVEIRA MJ, ARAÚJO WMC e BORGO LA, 2005).

Outros estudos também corroboram com a associação entre a incidência de câncer e a ingestão de carne processada evidenciando que as crianças que consomem esses embutidos em excesso podem ter riscos de desenvolver doenças cancerígenas ao longo da vida (HERRMANN SS, et al. 2015; BASTIDE NM et al. 2015).

Destaca-se também o alto consumo de biscoitos recheados pelos escolares. No estudo de Gomes VM, Santos MP e Freitas SM, (2010) foram analisados 15 recheios de biscoitos recheados de marcas diferentes, os quais possuíam em sua composição 55,4 a 76,9g de açúcar em uma proporção de 100 gramas, e mais de $50 \%$ da composição dos recheios se constituiu de açúcares simples, ou seja, uma alimentação abundante desses produtos ao longo do tempo se torna um gatilho para o aparecimento doenças crônicas não transmissíveis, com destaque para diabetes tipo 2, que possui ligação direta com o estilo de vida. Esses resultados corroboram com o presente estudo, com relação ao aumento da incidência de doenças crônicas como diabetes tipo 2 , em virtude do alto consumo de biscoitos recheados.

O suco industrializado ou de pacote está presente em um alto percentual nos recordatórios das crianças avaliadas. Estudo feito por Cardoso AMR, et al. (2013) mostra que o consumo de sucos industrializados pode causar a perda de minerais dentários, seja por cárie ou erosão dentária, pois o alto consumo de açúcar presentes nesses alimentos e na dieta como um todo favorece a adesão dos microorganismos aos dentes e o desenvolvimento da atividade cariogênica.

O consumo excessivo de produtos ultraprocessados pelas crianças em fase escolar revela uma alimentação rica em açúcares, sódio, gorduras trans e hidrogenadas, com baixo teor de fibras e minerais, aumentando as chances de desenvolverem doenças crônicas não transmissíveis, sobrepeso e obesidade, com carências nutricionais em vitaminas, minerais e fibras. Dessa forma, é essencial que se desenvolvam estratégias de educação nutricional nas escolas, para que alimentos inadequados ao consumo infantil não sejam inseridos nos lanches escolares e ao incentivo da prática de atividade física fora do ambiente escolar, visando em parte, uma prevenção para esse problema de saúde pública. Destaca-se ainda, a necessidade de mais pesquisas sobre os possíveis fatores determinantes do sobrepeso e obesidade nos escolares. 


\section{CONCLUSÃO}

O estado nutricional dos escolares mostrou um desenvolvimento de sobrepeso e obesidade e excesso de gordura abdominal que possui relação com riscos de doenças cardiovasculares. Além disso, há um alto consumo de alimentos ultraprocessados, com destaque para sucos de pacote, embutidos, bolos, biscoitos doces e recheados, levando a um aumento nos percentuais de carboidratos e lipídios do valor energético total da dieta dessas crianças. Esses hábitos alimentares estão relacionados a diversos prejuízos a saúde, entre eles, se destacam as doenças crônicas não transmissíveis, os problemas dentários e os cânceres. Sendo assim, fica evidente a necessidade de mais investimentos na área da nutrição e saúde, tornando-se imprescindível que haja um incentivo para uma alimentação saudável com base no guia alimentar e uma redução no consumo de alimentos ultraprocessados.

\section{REFERÊNCIAS}

1. ARAÚJO PF, RODRIGUES RS. Nitratos, nitritos, nitrosaminas e seus efeitos sobre o organismo humano. Revista Higiene Alimentar. 2008; 22(160):54-8.

2. ADAMI FS, CONDE SR. Alimentação e nutrição nos ciclos da vida. 1ำ edição, Lajeado, Rio Grande do Sul: Editora Univates, 2016.

3. BASTIDE NM et al. A central role for heme iron in colon carcinogenesis associated with red meat intake. Cancer Res, 2015; 75(5):870-879.

4. BERNARDI JR et al. Estimativa do consumo de energia e de macronutrientes no domicílio e naescolaem préescolares. Jornal de Pediatria,2010; 86(1): 59-64.

5. BRASIL. Resolução № 4 de abril de 2015, Dispõe a alteração da redação dos artigos 25 a 32 da Resolução/CD/FNDE no 26, de 17 de junho de 2013, no âmbito do Programa Nacional de 41 Alimentação Escolar (PNAE). Ministério da Educação. Fundo Nacional de Desenvolvimento da Educação. Conselho Deliberativo do Brasil. Brasília, DF, 8 de abr. 2015.

6. CARDOSO, Andreia Medeiros Rodrigues; SANTOS, Anderson Maikon de Souza; ALMEIDA, Flaubert Wesley Barbosa; ALBURQUERQUE, Tâmara Pereira de; XAVIER, Alidiane Fábia Cabral; CAVALCANTI, Alessandro Leite. Características Físico-Químicas de Sucos de Frutas Industrializados: Estudo in vitro. Revista Odonto, 2013 21(41/42): 9-17.

7. CHAVES C, LIMA F, MENDONÇA $F$ et al. Avaliação do crescimento e desenvolvimento de crianças institucionalizadas. Rev Bras Enferm. 2013 set-out; 66(5): 668-74.

8. Dietary Reference Intakes for energy, carbohydrate, fiber, fat, fatty acids, cholesterol, protein, and amino acids. Washington, D.C.: National Academy Press, 2002b.

9. DUTRA CB, RATH S, REYES FGR. Nitrosaminas voláteis em alimentos. Alim. Nutr. Araraquara, 2007; 18(1):111120.

10. FIDELIS CMF, OSÓRIO MF. Rev. Bras. Saúde Matern. Infant., Recife; jan - mar, 2007; 7(1): 63-74.

11. FREEDMAN DS et al. Relation of circumferences and skinfold thicknesses to lipid and insulin concentrations in children and adolescents: the Bogalusa Heart Study. Am J Clin Nutr. 1999;69: 308-17.

12. GUEDES D, ALMEIDA F, NETO J et al. Baixo peso corporal/magreza, sobrepeso e obesidade de crianças e adolescentes de uma região brasileira de baixo desenvolvimento economic. Rev Paul Pediatr 2013;31(4):437-43.

13. GOMES VM, SANTOS MP, LEMOS FREITAS SM. Análise de açucares e gorduras de recheios em biscoitos recheado sabor chocolate. Ver. Ceres, 2010; 5(1); 19-25.

14. GUERREIRO RS, SÁ MS, RODRIGUES LAP. Avaliação do teor de nitrito e nitrato em alimentos cárneos comercializados em Salvador. Rev Inter. 2012;5(1):77-91.

15. HERRMANN SS et al. Dietary exposure to volatile and non-volatile N-nitrosamines from processed meat products in Denmark. Food Chem Toxicol, 2015; 16(80):137-143.

16. MIRANDA JMQ et al. Prevalência de sobrepeso e obesidade infantil em instruções de ensino: públicas vs. privadas. Rev. Bras Med Esporte - Vol 21, n-2 - Mar-Abr, 2015.

17. OLIVEIRA MJ, ARAÚJO WMC, BORGO LA. Quantificação de nitrito e nitrato em linguiças do tipo frescal. Ciênc Tecnol Aliment.2005;25(4):736-42.

18. ROCHA LM. Obesidade infantil: uma revisão bibliográfica. Trabalho de conclusão de curso (Curso de especialização em estratégia saúde da família) - Universidade Federal de Minas Gerais, Belo Horizonte - Minas Gerais, 2013.

19. RODRIGUES CARDOSO AM et al. Características Físico-Quimicas de sucos de frutas industrializados: Estudo in vitro. Rev. Odonto 2013; 21(41-42): 9-17.

20. SANTOS DM. A alimentação escolar como estratégia de educação alimentar e nutricional: uma revisão da literatura. Trabalho de conclusão de curso (Curso de graduação em Nutrição) - Universidade Federal de Pernambuco, Vitória de Santo Antão, 2017.

21. SILVA J, TIMÓTEO A, SANTOS $C$ et al. Consumo alimentar de crianças e adolescentes residentes em uma área de invasão em Maceió, Alagoas, Brasil. Rev Bras Epidemiol 2010; 13(1): 83-93.

22. SOARES LR et al A transição da desnutrição para a obesidade. Brazilian Journal of Surgery and Clinical, [s.I.], 2013Dez/Fev 2014;5(1):64-68. 